\title{
Shape Features based Apple and Banana Fruit Image Classification
}

\author{
D.O.I- 10.51201/12461 \\ https://doi.org/10.51201/12461 \\ Manjunath Ravikumar ${ }^{1}$, Sharanabasappa ${ }^{2}$ and ${ }^{3}$ RaghavendraAnantayya \\ ${ }^{1}$ Department of Computer Science \\ Sri. Shivalingeshwar Governement First Grade Degree College \\ Madanhipparga, Tq.Aland, Dist. Kalaburagi, Karnataka, India \\ ${ }^{2}$ Department of Computer Science \\ Sharanabasaveshwar College of Science, Kalaburagi, Karnataka, India \\ ${ }^{3}$ Department of Computer Science \\ Gulbarga University, Kalaburagi, Karnataka, India
}

\begin{abstract}
This paper presents the classification of Apple and Banana fruits by using images. Detection of fruits is a challenging task. Choosing the desired fruits from many other fruits task is cumbersome, so to eliminate this difficulty this experiment is carried out. This system works in three stages first image preprocessing, second, extraction of relevant features and at last classification. The fruit-360 dataset has taken for the images of fruits. The shape features are extracted from the input images of Apple and Banana. Finally, the extracted features were supplied as input to the most five popular classifiers; LDA, Nä̈ve Bays, Logistic regression, SVM and KNN. The KNN classifier has given highest as $99.10 \%$ classification accuracy among other classifiers.
\end{abstract}

Key words: Shape features, LDA, KNN, SVM, Naïve bays Logistic_regression.

\section{Introduction}

It is a recurring activity in supermarkets to identify various kinds of fruits, where the cashier must be able to find out not just the species of a specific fruit (i.e. banana, apple) but also its variety, which will decide its price (Rocha et al., 2010). Because of advancements in computers and microelectronics related to conventional imaging, optical image analysis and imageprocessing technologies help to circumvent these concerns(Dubey \& Jalal, 2015). Work has been carried out in the field of image analysis and labeling of fruit and vegetables to help work in the field of agricultural robotics and robotic fruit harvesting (Nosseir \& Ashraf Ahmed, 2019). Nandhini and Jaya (Nandhini. P.and J. Jaya.2014), compared the CBBIS Color Dependent Binary Image Segmentation and Particle Swarm Optimization PSO techniques. They have categorised pineapple, tomato, squash, and onion. (Linker et al., 2012) attempted to detect green "Golden Delicious" apple fruit from RGB images on only part of the apple tree to promote the 
study; the algorithm detected more than 85 percent of the apples shown in the images below natural lighting ( Linker et al., 2012). Hence, the fruit classification(Sakib \& Ashrafi, 1980),(Nosseir \& Ashraf Ahmed, 2019),(Nayak, 2019),(Rocha et al., 2010) work is undertaken for the proposed work.

\section{Dataset}

To carry out this experiment the dataset named as Fruit-360(Sakib \& Ashrafi, 1980) has been used. The dataset contains many fruits images. Among all fruit images we have considered only Apple and Banana images. The 1k Apple fruit and 1k Banana images were taken.

Following figure shows the sample input images.

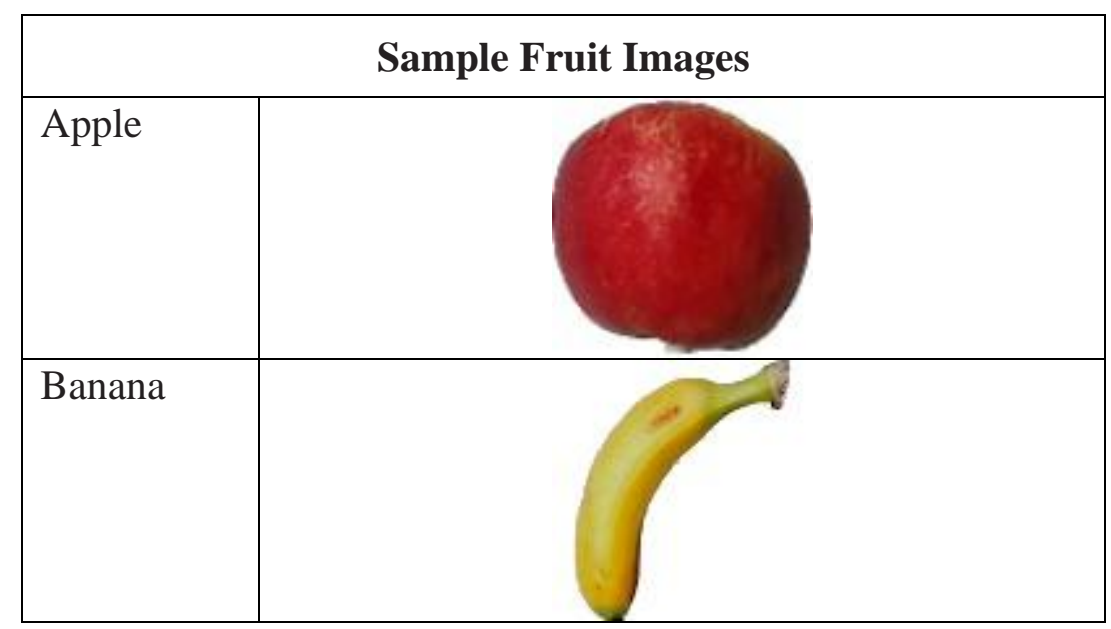

Figure 1: Sample input image

\section{Proposed method}

This method have considered the shape features. The shape characteristics or visual characteristics are called visual features of objects. For instance, circular or triangular objects or other shapes, the object's perimeter boundary, the border diameter, and so on (Liu \& Shi, 2011). The following algorithm shows the feature extraction and classification of Apple and Banana fruits.

Algorithm: Apple and Banana Fruit classification

Input: Fruit image

Output: Classified fruit

Start:

Step 1 : Read the input image 
Step 2: Apply histogram equalization method on input image

Step 3: Convert RGB image into Grayscale image

Step 4: Convert Grayscale image into binary image.

Step 5: From binary image extract shape features (MajorAxisLength,MinorAxisLength Centroid, Eccentricity, EulerNumber, Orientation, Perimeter, Slidity)

Step 6: Store the extracted features in knowledge base alongwith its labels

Step 7: Set 5 fold cross-validation and apply the classifiers like (LDA,NB,Logistic Regression, SVM and $\mathrm{KNN}$ )

Step 8: Display the classified fruit

Stop.

\section{Experimental Result}

To carry out the experiment the standard dataset named as fruit-360. Five popular classifiers are evaluated to obtain the classification accuracy. The following table shows the performance.

Table 1: Classification accuracy of five classifiers.

\begin{tabular}{|r|l|c|c|}
\hline \multicolumn{4}{|c|}{ Apple and Banana Classification Accuracy } \\
\hline $\begin{array}{c}\text { Sl. } \\
\text { No. }\end{array}$ & Classifier & Rec.Acc. & $\begin{array}{c}\text { Training } \\
\text { Time in } \\
\text { (Sec) }\end{array}$ \\
\hline 1 & LDA & $85.00 \%$ & 10.292 \\
\hline 2 & Naïve_Bays & $87.00 \%$ & 2.2948 \\
\hline 3 & Logistic_regression & $93.60 \%$ & 12.077 \\
\hline 4 & SVM & $93.90 \%$ & 4.766 \\
\hline 5 & KNN & $\mathbf{9 9 . 1 0 \%}$ & $\mathbf{2 . 2 9 4 8}$ \\
\hline
\end{tabular}




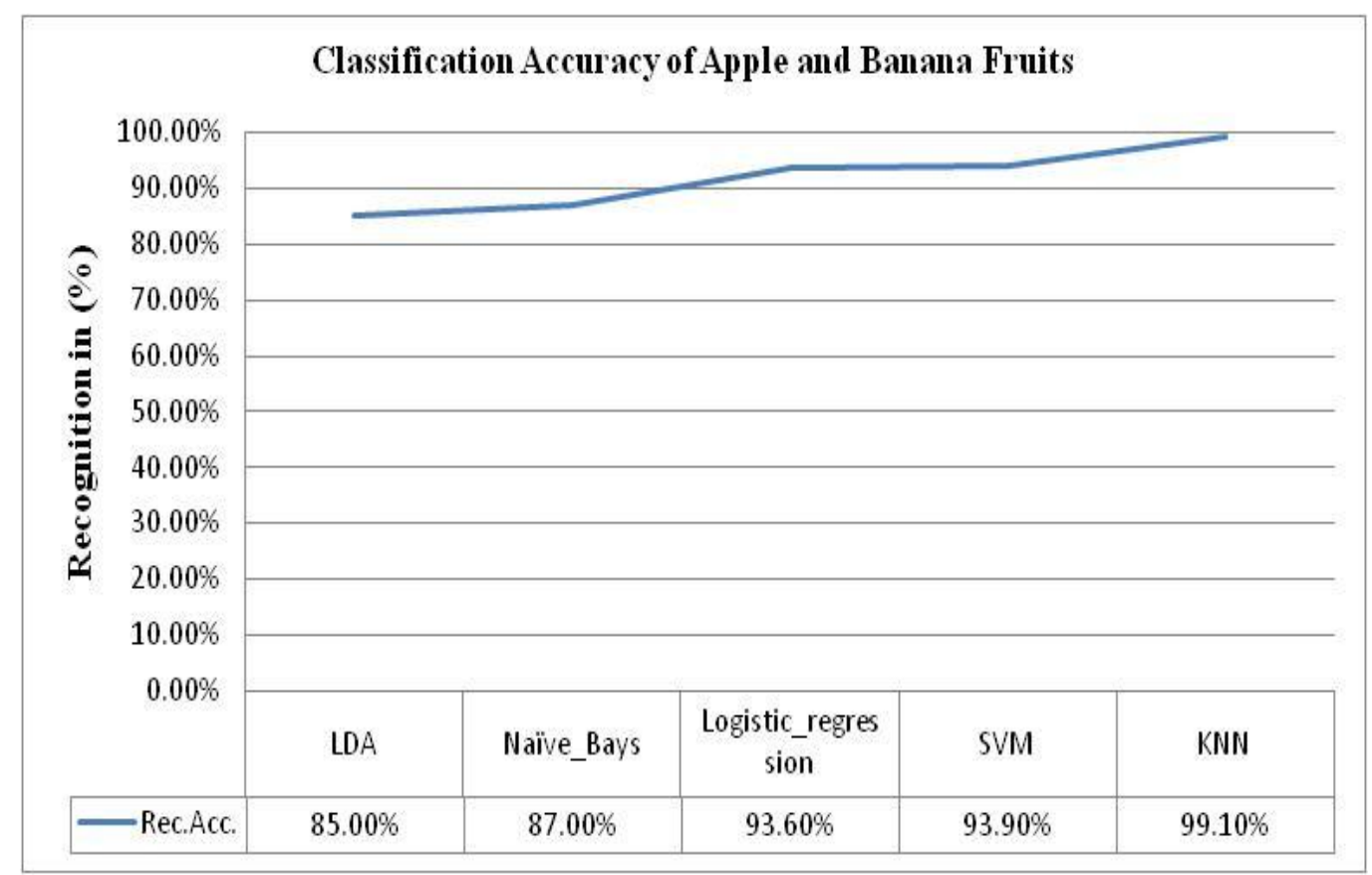

Figure 1: Performance of the classifiers over the shape features.

The below figures shows the individual performance of each classifiers over the shape features extracted from the Apple and Banana fruit.

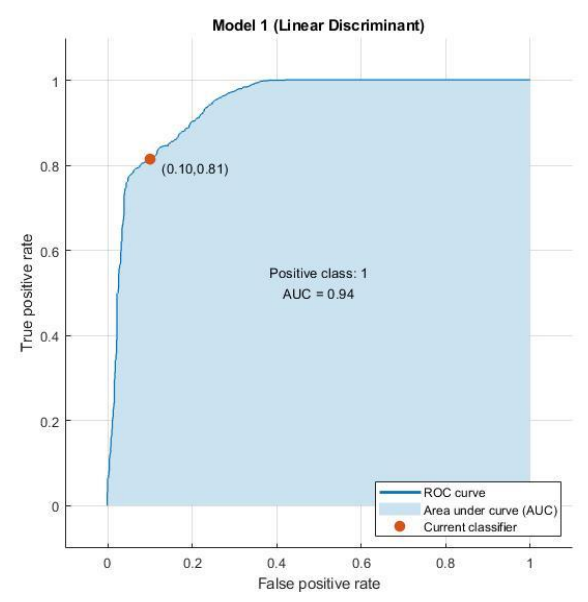

Figure 2: ROC of LDA Classifier. 


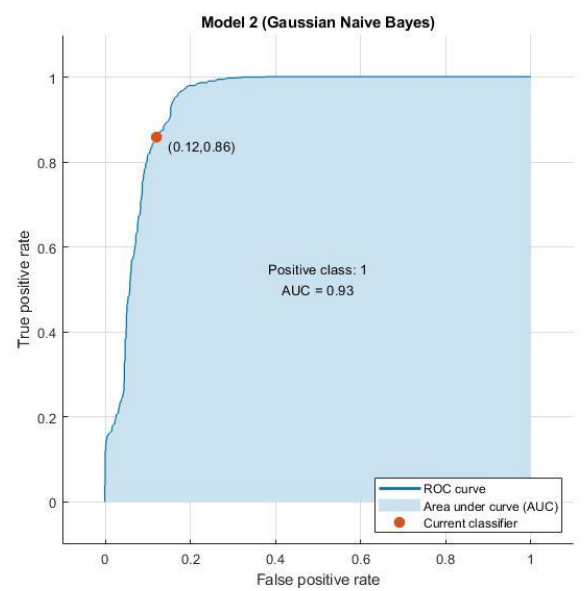

Figure 3: ROC of Naivebays classifier.

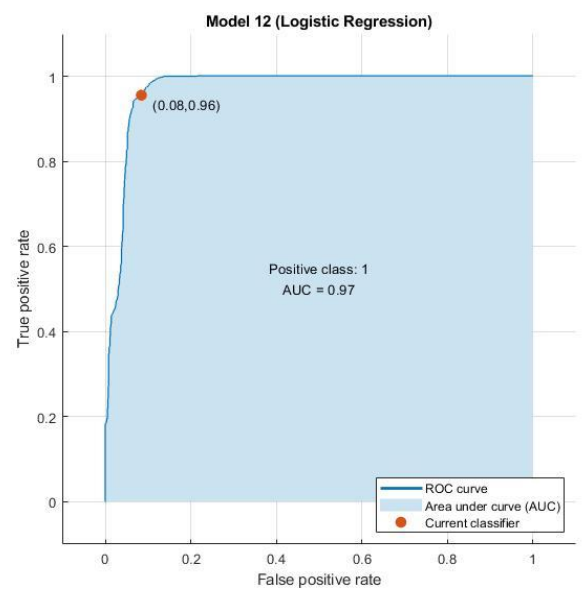

Figure 4: ROC for Logistic Regression

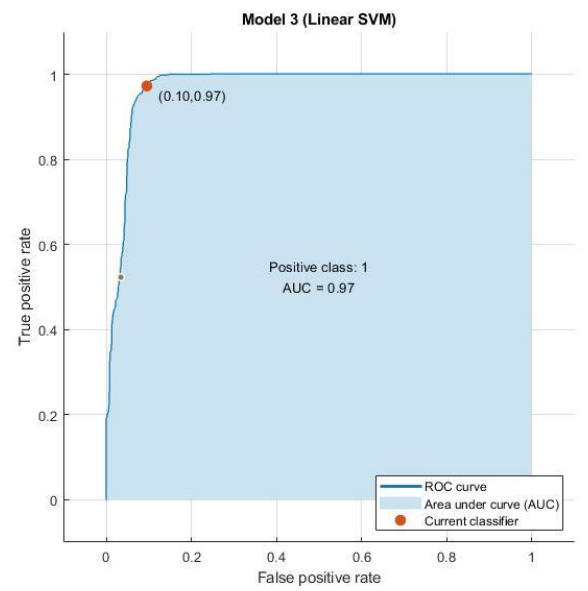

Figure 5: ROC for SVM 


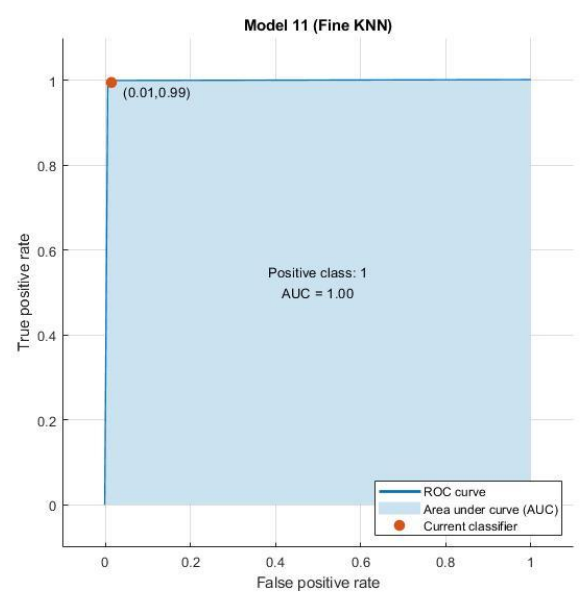

Figure 6: ROC for KNN Classifier

From the above table and graph it is clearly observed that the KNN classifier has outperformed from other classifiers.

\section{Conclusion}

The classification of Apple and Banana fruit were carried out in this proposed method. The shape feature has used as the features. The five classifiers namely, LDA, Naïve bays, Logistic Regression, SVM and KNN. In the future work the other features will considered and those features should less in numbers and high in classification accuracy.

\section{References}

1. Dubey, S. R., \& Jalal, A. S. (2015). Application of Image Processing in Fruit and Vegetable Analysis: A Review. Journal of Intelligent Systems, 24(4), 405-424. https://doi.org/10.1515/jisys-2014-0079

2. Liu, J., \& Shi, Y. (2011). Image feature extraction method based on shape characteristics and its application in medical image analysis. Communications in Computer and Information Science, 224 CCIS(PART 1), 172-178. https://doi.org/10.1007/978-3-64223214-5_24

3. Nayak, M. A. M. (2019). Fruit Recognition using Image Processing. 7(08), 1-6.

4. Nosseir, A., \& Ashraf Ahmed, S. E. (2019). Automatic classification for fruits' types and identification of rotten ones using k-NN and SVM. International Journal of Online and Biomedical Engineering, 15(3), 47-61. https://doi.org/10.3991/ijoe.v15i03.9832

5. Rocha, A., Hauagge, D. C., Wainer, J., \& Goldenstein, S. (2010). Automatic fruit and vegetable classification from images. 70, 96-104. https://doi.org/10.1016/j.compag.2009.09.002 
6. Sakib, S., \& Ashrafi, Z. (1980). Implementation of Fruits Recognition Classifier using Convolutional Neural Network Algorithm for Observation of Accuracies for Various Hidden Layers. 8-11.

7. G. G. Rajput Suryakanth Baburao Ummapure (2019). Multi-Feature based Handwritten Script Identification at word level. International Journal of Innovative Technology and Exploring Engineering (IJITEE). 9(2), pp. 3896-3901

8. G.G Rajput, Suryakanth Baburao Ummapure (2019). Script Identification from Handwritten document Images Using LBP Technique at Block level. 2019 International Conference on Data Science and Communication (IconDSC). IEEE.

9. Suryakanth Baburao Ummapure G. G. Rajput. (2018). Script Identification from Handwritten Document Images Using LBP features. International Journal of Computational Engineering Research (IJCER). 8(9). pp. 13-21

10. Satish Kumar, Raghavendra Srinivas (2013). A study on Image Segmentation and its Methods. International Journal of Advanced Research in Computer Science and Software Engineering. 3(9). pp. 1112-1114. 